Proyecciones

Vol. 19, $\mathrm{N}^{o}$ 1, pp. 53-63, May 2000

Universidad Católica del Norte

Antofagasta - Chile

\title{
CORPS DE NOMBRES D'INDICE DIVISIBLE PAR UN ENTIER DONNÉ, DANS FACTEUR CARRÉ
}

\author{
DENIS HÉMARD \\ Univ. de Valenciennes et du Hainaut Cambrésis, \\ France
}

\begin{abstract}
If $K$ is a number field, $\mathcal{O}_{K}$ its ring of integers and $\alpha \in \mathcal{O}_{K}$ such that $K=\mathbf{Q}(\alpha)$, the index of $\alpha$ is the index of abelian groups ind $\alpha=\left(\mathcal{O}_{K}: \mathbf{Z}[\alpha]\right) \in \mathbf{N}$ and the index $i(K)$ of $K$ is the gcd of these ind $\alpha$. We show a way to construct via Hilbert class fields of quadratic number fields, for any integer $n$ without square factor, an infinite family of number fields $K$ with $i(K)$ divisible by $n$.
\end{abstract}




\section{Introduction}

Le paragraphe 1 présente une variante de l'interprétation habituelle de la notion d'indice, qui permet une démonstration simplifiée d'un théorème de Dedekind et d'un de ses corollaires (corollaire 2) (on pourra consulter $[\mathrm{N}]$ ou $[\mathrm{H}]$ pour une autre présentation).

Le paragraphe 2 se base sur ce corollaire pour construire des corps de nombres d'indice divisible par un entier donné sans facteur carré. On les obtient comme sous-corps (laissés fixes par certains sous-groupes de Galois) de corps de classes de Hilbert de certaines extensions quadratiques de Q. Et un résultat de Yamamoto $[\mathrm{Y}]$ nous assure l'existence d'une infinité de telles extensions quadratiques.

Le fait qu'il y ait une infinité de corps de nombres d'indice divisible par un entier donné sans facteur carré était déjà connu de M. Bauer en 1907 ([B]). Sa méthode basée sur les polygones de Newton et le corollaire déjà cité, fournit des polynômes irréductibles définissant de telles extensions. Il nous a semblé utile d'en rappeler le principe dans un appendice. (Voir également, pour d'autres résultats sur l'indice, les références données dans [N] p198)

\section{Le théorème de Dedekind}

Soient $K$ un corps de nombre de degré $n$ sur $\mathbf{Q}, \mathcal{O}_{K}$ son anneau d'entiers et $\alpha \in \mathcal{O}_{K}$ tel que $K=\mathbf{Q}(\alpha)\left(\simeq \frac{\mathbf{Q}[X]}{(P)}\right.$ où $P=\operatorname{Irr}(\alpha, \mathbf{Q}) \in$ $\mathbf{Z}[X]$ est le polynôme minimal de $\alpha$ sur $\mathbf{Q})$. On sait que $\mathcal{O}_{K}$ est un $\mathbf{Z}$-module libre de rang $n$ et $\mathbf{Z}[\alpha] \simeq \frac{\mathbf{Z}[X]}{(P)}$ un sous $\mathbf{Z}$-module libre de même rang; d'où la notion d'indice :

ind $\alpha=\left|\frac{\mathcal{O}}{\mathbf{Z}[\alpha]}\right|=\left(\mathcal{O}_{K}: \mathbf{Z}[\alpha]\right)=\prod_{i=1}^{r} m_{i}$ où $m_{r}\left|m_{r-1}\right| \ldots \mid m_{1}$ sont les facteurs invariants $(\neq 1)$ du sous $\mathbf{Z}$-module $\mathbf{Z}$ de $\mathcal{O}_{K}$.

\section{Définition :}

On appelle indice de $K / \mathbf{Q}$ l'entier $i(K)=\operatorname{pgcd}\left\{\right.$ ind $\alpha ; \quad \alpha \in \mathcal{O}_{K}$, $\mathbf{Q}(\alpha)=K\}$. 


\section{Remarque :}

En choisissant une Z Z-base $\left(e_{1}, \ldots, e_{n}\right)$ de $\mathcal{O}_{K}$ de telle sorte que $\left(m_{1} e_{1}, \ldots, m_{n} e_{n}\right)$ soit une $\mathbf{Z}$-base de $\mathbf{Z}[\alpha]\left(\right.$ avec $\left.m_{r+1}=\cdots=m_{n}=1\right)$ , on voit que les discriminants vérifient l'égalité :

$$
(\operatorname{ind} \alpha)^{2} \operatorname{disc}\left(\mathcal{O}_{K} / \mathbf{Z}\right)=\operatorname{disc}(\mathbf{Z}[\alpha] / \mathbf{Z}) .
$$

D'où le fait que les diviseurs de l'indice $i(K)$ soient généralement désignés en allemand, par "außerwesentliche Diskriminantenteiler" et en anglais, par "(common) inessential discriminantal divisor" (ils divisent tous les $\operatorname{disc}(\mathbf{Z}[\alpha] / \mathbf{Z})$ sans nécessairement diviser $\left.\operatorname{disc}\left(\mathcal{O}_{K} / \mathbf{Z}\right)\right)$.

Après tensorisation par $\mathbf{F}_{p}=\mathbf{Z} / p \mathbf{Z}(p$ premier $\in \mathbf{N})$, l'isomorphisme $\frac{\mathbf{Z}[X]}{(P)} \simeq \mathbf{Z}[\alpha]$ et l'injection canonique $\mathbf{Z}[\alpha] \hookrightarrow \mathcal{O}_{K}$ fournissent les morphismes

$$
\frac{\mathbf{F}_{p}[X]}{(\bar{P})} \simeq \frac{\mathbf{Z}[\alpha]}{p \mathbf{Z}[\alpha]} \stackrel{\varphi_{\alpha}}{\longrightarrow} \frac{\mathcal{O}_{K}}{p \mathcal{O}_{K}}
$$

de $\mathbf{F}_{p}$-algèbres de même $\mathbf{F}_{p}$-dimension $n$ (où $\bar{P}=$ image de $P$ dans $\left.\mathbf{F}_{p}[X]\right)$.

\section{Lemme :}

a) $p \nmid i n d \alpha \Leftrightarrow \varphi_{\alpha}$ est un isomorphisme.

b) $p \nmid i(K) \Leftrightarrow \frac{\mathcal{O}_{K}}{p \mathcal{O}_{K}}$ est une $\mathbf{F}_{p}$-algèbre monogène.

\section{Démonstration :}

a) $p \nmid i n d \alpha \Leftrightarrow \frac{\mathcal{O}_{K}}{\mathbf{Z}[\alpha]} \otimes \mathbf{F}_{p}=0 \Leftrightarrow \varphi_{\alpha}$ surjectif

$\Leftrightarrow \varphi_{\alpha}$ isomorphisme (par égalité des $\mathbf{F}_{p}$-dimensions)

b) $p \nmid i(K) \Leftrightarrow \exists \alpha \in \mathcal{O}_{K}, \mathbf{Q}(\alpha)=K$ et $\varphi_{\alpha}$ isomorphisme (par a))

$\Leftrightarrow \mathcal{O}_{K} / p \mathcal{O}_{K} \mathbf{F}_{p}$-algèbre monogène (engendrée par $\alpha \bmod p \mathcal{O}_{K}$ ).

(Pour le sens $\Leftarrow$, on remarque que si les $n$ premières puissances de $\alpha$ sont $\mathbf{F}_{p}$-linéairement indépendantes modulo $p \mathcal{O}_{K}$, on a $\operatorname{deg}(\operatorname{Irr}(\alpha, \mathbf{Q}))=$ $n$ et donc $\mathbf{Q}(\alpha)=K)$.

\section{Corollaire 1 (Théorème de Dedekind) :}

Si $p \nmid i n d \alpha$, soit $\bar{P}=\prod_{i=1}^{s} \bar{Q}_{i}^{r_{i}}$ la décomposition en irréductibles unitaires de l'image $\bar{P}$ de $P=\operatorname{Irr}(\alpha, \mathbf{Q})$ dans $\mathbf{F}_{p}[X]$ (et les $Q_{i}$ unitaires, antécédents de $\bar{Q}_{i}$ dans $\left.\mathbf{Z}[X]\right)$; alors 
a) les idéaux de $\mathcal{O}_{K}, p_{i}=\left(p, Q_{i}(\alpha)\right)$, sont les idéaux premiers de $\mathcal{O}_{K}$ au dessus de $p$; il y en a donc $s$.

b) $\operatorname{deg} Q_{i}=f\left(p_{i} / p\right)$ (le degré résiduel)

c) $r_{i}=e p_{i} / p$ (l'indice de ramification) pour tout $i \in\{1, \ldots, s\}$.

(En particulier, si $p \nmid i(K)$, la décomposition de $p$ dans $\mathcal{O}_{K}$ est complètement déterminée par la connaissance d'un $\alpha$ tel que $p \nmid i n d \alpha$.)

\section{Démonstration :}

Par hypothèse, l'application

$$
\begin{gathered}
\frac{\mathbf{F}_{p}[X]}{(\bar{P})} \longrightarrow \frac{\mathcal{O}_{K}}{p \mathcal{O}_{K}} \\
\bar{Q} \bmod (\bar{P})^{\longmapsto} \longmapsto Q(\alpha) \operatorname{modp} \mathcal{O}_{K}
\end{gathered}
$$

(indépendante du choix de l'antécedent $Q \in \mathbf{Z}[X]$, de $\bar{Q}$ )

est un isomorphisme de $\mathbf{F}_{p}$-algèbres.

On en déduit une correspondance bijective entre idéaux premiers de $\mathbf{F}_{p}[X]$ contenant $\bar{P}$ et idéaux premiers de $\mathcal{O}_{K}$ contenant $p$; d'où a) puisque les idéaux premiers de $\mathbf{F}_{p}[X]$ contenant $\bar{P}$ sont les $\left(\bar{Q}_{i}\right), i=$ $1, \ldots, s$. b) s'en déduit car

$$
\operatorname{deg} \bar{Q}_{i}=\operatorname{dim} \frac{\mathbf{F}_{p}[X] /(\bar{P})}{\left(\bar{Q}_{i}\right) /(\bar{P})}=\operatorname{dim} \frac{\mathcal{O}_{K} / p \mathcal{O}_{K}}{\left(Q_{i}(\alpha), p\right) / p \mathcal{O}_{K}}=f\left(p_{i} / p\right)
$$

ainsi que c), car dans $\frac{\mathbf{F}_{p}[X]}{(\bar{P})}$, il y a exactement $r_{i}+1$ puissances distinctes de l'idéal engendré par $\bar{Q}_{i} \bmod (\bar{P})$ (correspondant aux exposants $\left.0,1, \ldots, r_{i}\right)$.

\section{Corollaire 2 :}

Soit $p \mathcal{O}_{K}=p_{1}^{e_{1}} \ldots p_{s}^{e_{s}}$ la décomposition dans $\mathcal{O}_{K}$ d'un $p$ premier $\in \mathbf{N}$ et $f_{i}=f\left(p_{i} / p\right)$, pour $i=1, \ldots, s$; alors

$$
\begin{gathered}
p \dagger i(K) \Leftrightarrow \exists P_{1}, \ldots, P_{s} \in \mathbf{F}_{p}[X], \text { irréductibles, unitaires, distincts, } \\
\text { de degré } f_{1}, \ldots, f_{s} \text { respectivement. }
\end{gathered}
$$




\section{Démostration :}

$\Rightarrow): \exists \alpha \in \mathcal{O}_{K}, p \nmid i n d \alpha$; on applique alors le corollaire 1 .

$\Leftarrow)$ : Comme extensions (de corps) de même degré sur $\mathbf{F}_{p}$, on a des isomorphismes de $\mathbf{F}_{p}$-algèbres :

$$
\frac{\mathcal{O}_{K}}{p_{i}} \simeq \frac{\mathbf{F}_{p}[X]}{\left(P_{i}\right)} \quad \forall i=1, \ldots, s .
$$

Par un petit lemme (démontré ci-dessous), on en déduit des isomorphismes de $\mathbf{F}_{p}$-algèbres :

$$
\frac{\mathcal{O}_{K}}{p_{i}^{e_{i}}} \simeq \frac{\mathbf{F}_{p}[X]}{\left(P_{i}^{e_{i}}\right)} \quad \forall i=1, \ldots, s .
$$

Par application du théorème chinois, il en résulte des isomorphismes de $\mathbf{F}_{p}$-algèbres :

$$
\frac{\mathbf{F}_{p}[X]}{\left(P_{1}^{e_{1}} \ldots P_{s}^{e_{s}}\right)} \simeq \prod_{i=1}^{s} \frac{\mathbf{F}_{p}[X]}{\left(P_{i}^{e_{i}}\right)} \simeq \prod_{i=1}^{s} \frac{\mathcal{O}_{K}}{p_{i}^{e_{i}}} \simeq \frac{\mathcal{O}_{K}}{p \mathcal{O}_{K}} .
$$

En particulier $\frac{\mathcal{O}_{K}}{p \mathcal{O}_{K}}$ est une $\mathbf{F}_{p}$-algèbre monogène et donc $p \nmid i(K) . \diamond$

\section{Démonstration du lemme utilisé :}

$$
\frac{\mathbf{F}_{p}[X]}{(P)} \simeq \frac{\mathcal{O}_{K}}{p} \Rightarrow \frac{\mathbf{F}_{p}[X]}{\left(P^{r}\right)} \simeq \frac{\mathcal{O}_{K}}{p^{r}} \quad \forall r=1, \ldots, e
$$

(on a omis les indices i).

Soit $R$ un relèvement unitaire de $P$, dans $\mathbf{Z}[X]$; on peut choisir $\alpha \in$ $\mathcal{O}_{K}$ tel que $R(\alpha) \in p \backslash p^{2}$. Alors $P^{r-1}\left(\alpha \bmod p^{r}\right)=R^{r-1}(\alpha) \bmod p^{r} \neq 0$. Par conséquent, l'homomorphisme de $\mathbf{F}_{p}[X]$ dans $\mathcal{O}_{K} / p^{r}$ qui envoie $X$ sur $\alpha \bmod p^{r}$ est de noyau $\left(P^{r}\right)$ et induit donc l'isomorphisme cherché, par égalité des $\mathbf{F}_{p}$-dimensions (égalité qu'on peut vérifier par une récurrence et un "dévissage" après avoir localisé $\mathcal{O}_{K} / p^{r}$ en $p$, ce qui ne change rien à ce quotient mais permet de supposer $\mathcal{O}_{K}$ principal). 


\section{Remarque :}

On utilisera ce corollaire sous la forme contraposée équivalente (avec les notations supplémentaires $r\left(f_{i}\right)=\#\left\{p_{i} \mid p, f\left(p_{i} / p\right)=f_{i}\right\}$ et $g\left(f_{i}\right)=\#\left\{P \in \mathbf{F}_{p}[X]\right.$, unitaires, irréductibles et de degré $\left.\left.f_{i}\right\}\right)$ :

$$
p \mid i(K) \Leftrightarrow \exists i \in\{1, \ldots, s\} \quad r\left(f_{i}\right)>g\left(f_{i}\right)
$$

\section{Corps de nombres d'indice divisible par un en- tier donné sans facteur carré}

Soit $k$ une extension galoisienne finie de $\mathbf{Q}$ et $H$ l'extension abélienne de $k$ partout non ramifiée, de degré impair, maximale (dans une clôture algébrique de $\mathbf{Q})$. On notera $\Gamma=\operatorname{Gal}(H / k)$ d'ordre $|\Gamma|=m$ (impair) et $C l_{k}^{\prime}=C l_{k} / C l_{k}(2)$ le quotient du groupe des classes d'idéaux de $k$ par sa partie 2-primaire.

Par maximalité $H / \mathbf{Q}$ est galoisienne et le corps de classes fournit un isomorphisme de $\operatorname{Gal}(k / \mathbf{Q})$-modules $C l_{k}^{\prime} \simeq \Gamma$ (qui à la classe $\bar{p}$ d'un idéal premier $p$ associe son Frobenius Frob $p$ dans $\Gamma$ ).

Prenons pour $k$ une extension quadratique de $\mathbf{Q}$. Alors les 2-Sylow de $\operatorname{Gal}(H / \mathbf{Q})$ sont d'ordre 2 et n'importe quel élément $\sigma$ d'ordre 2 dans $\operatorname{Gal}(H / \mathbf{Q})$ est un relèvement du générateur $\tau$ de $\operatorname{Gal}(k / \mathbf{Q})$. En particulier $\operatorname{Gal}(H / \mathbf{Q})=\Gamma \times \operatorname{Gal}(k / \mathbf{Q})$. Soit $K=H^{<\sigma>}$ le corps laissé fixe ; alors :

\section{Proposition :}

Avec ces notations, pour $p$ premier $\in \mathbf{N}$ et $m$ la partie impaire du nombre de classes de $k$, on a $p \mid i(K)$ dans les cas suivants :

a) $p$ ramifié dans $k / \mathbf{Q}$ et $m>2 p-1$.

b) $p$ inerte dans $k / \mathbf{Q}$ et $m>p^{2}-p+1$.

\section{Démonstration :}

Comme $\tau$ opère par inversion sur $C l_{k}$ et $C l_{k}^{\prime}$ (en particulier toute sous-extension de $H / k$ reste galoisienne sur $\mathbf{Q}$ ), on a

$$
\gamma \sigma \gamma \sigma=\gamma \gamma^{-1}=1 \quad \forall \gamma \in \Gamma
$$


Par conséquent, il y a $m$ éléments d'ordre 2 et donc $m$ 2-Sylow dans $\operatorname{Gal}(H / \mathbf{Q})$, lesquels sont conjugés par les théorèmes de Sylow ; ce sont donc les ${ }^{\gamma}<\sigma>, \gamma \in \Gamma$ (où $\gamma \sigma=\gamma \sigma \gamma^{-1}$ ).

Si $p$ ramifié dans $k / \mathbf{Q}: p \mathcal{O}_{k}=p^{2} \Rightarrow \bar{p}=1$ dans $C l_{k}^{\prime} \Rightarrow$ Frob $p=1$ dans $\Gamma \Rightarrow p \mathcal{O}_{H}=\mathcal{B}_{1} \ldots \mathcal{B}_{m}=\prod_{\gamma \in \Gamma} \gamma(\mathcal{B})$ totalement décomposé.

Le groupe d'inertie sur $\mathbf{Q}$ de $\mathcal{B}=\mathcal{B}_{1}$ est de la forme $T_{\mathcal{B}}=<\sigma>$ d'ordre 2. Ses conjugués sont les ${ }^{\gamma} T_{\mathcal{B}}=T_{\gamma(\mathcal{B})}$; ils sont tous distincts : ce sont les $m$ 2-Sylow de $\operatorname{Gal}(H / \mathbf{Q})$. Donc si $K=H^{<\sigma>}$ est le corps laissé fixe par $\sigma$, on a :

$$
\operatorname{Gal}(H / K)=T_{\mathcal{B}} \Rightarrow \mathcal{B}^{2}=\wp_{1} \mathcal{O}_{H} \text { pour un } \wp_{1} \mid p \text { dans } \mathcal{O}_{K}
$$

et pour $2 \leq i \leq m$,

$$
T_{\mathcal{B}_{i}} \cap T_{\mathcal{B}}=\{1\} \Rightarrow \exists j \neq i \text { et } \wp_{i} \mid p \text { dans } \mathcal{O}_{K}, \quad \mathcal{B}_{i \mathcal{B} j}=\wp_{i} \mathcal{O}_{H}
$$

(on numérotera les $\mathcal{B}_{i}, 2 \leq i \leq m$, de sorte que les $\frac{m-1}{2}$ premiers s'associent aux $\frac{m-1}{2}$ derniers).

D'où

$$
p \mathcal{O}_{K}=\wp_{1} \wp_{2}^{2} \ldots \wp_{\frac{m+1}{2}}^{2} .
$$

Il y a donc $\frac{m+1}{2}$ idéaux premiers de $\mathcal{O}_{K}$ au-dessus de $p$, dont les degrés résiduels sont tous égaux à 1 .

Il suffit donc que $\frac{m+1}{2}>p$ c'est à dire $m>2 p-1$ pour que $p \mid i(K)$ par le corollaire 2 de $\S 1$.

$\underline{\text { Si } p \text { inerte dans }} k / \mathbf{Q}: p \mathcal{O}_{k}=p$; on a comme précédemment

$$
p \mathcal{O}_{H}=\mathcal{B}_{1} \ldots \mathcal{B}_{m}=\prod_{\gamma \in \Gamma} \gamma(\mathcal{B}) \text {. }
$$

Le groupe de décomposition sur $\mathbf{Q}$ de $\mathcal{B}=\mathcal{B}_{1}$ est de la forme $D_{\mathcal{B}}=<$ $\sigma>$ d'ordre 2 , distincts des autres $D_{\mathcal{B}_{i}}$ qui lui sont conjugués.

$$
K=H^{<\sigma>} \Rightarrow \operatorname{Gal}(H / K)=D_{\mathcal{B}} \Rightarrow \begin{array}{r}
\exists \wp_{1} \mid p \text { dans } \mathcal{O}_{K} \\
\wp_{1} \mathcal{O}_{K}=\mathcal{B}
\end{array}
$$

et pour $2 \leq i \leq m$,

$$
D_{\mathcal{B}_{i}} \cap D_{\mathcal{B}}=\{1\} \Longrightarrow \exists j \neq i \text { et } \wp_{1} \mid p \text { dans } \mathcal{O}_{K}, \mathcal{B}_{i \mathcal{B} j}=\wp_{1} \mathcal{O}_{H} .
$$

D'où

$$
p \mathcal{O}_{K}=\wp_{1} \wp_{2} \ldots \wp_{\frac{m+1}{2}}
$$

avec $f\left(\wp_{1} / p\right)=1, f\left(\wp_{1} / p\right)=2 \quad \forall i=2, \ldots \frac{m+1}{2}$. 
Comme le nombre de polynômes irréductibles unitaires et de degré

2 sur $\mathbf{F}_{p}$ est $\frac{p^{2}-p}{2}$, il suffit donc que $\frac{m-1}{2}>\frac{p^{2}-p}{2}$ c'est à dire $m>$ $p^{2}-p+1$ pour que $p \mid i(K)$ par le corollaire 2 de $\S 1$.

\section{Remarque :}

$H$ est la clôture galoisienne de $K$ et $k$ est l'unique extension quadratique de $\mathbf{Q}$, contenue dans $H$ ( $\operatorname{car} 4 \dagger|\operatorname{Gal}(H / \mathbf{Q})|)$. Deux extensions quadratiques distinctes de $\mathbf{Q}$ ne peuvent donc fournir la même extension $K$.

\section{Corollaire :}

Pour un entier $n=p_{1} \ldots p_{r}$ sans facteur carré, il y a une infinité de corps de nombres d'indice divisible par $n$.

\section{Démonstration :}

Par un résultat de Yamamoto ([Y], théorèmes 1 et 2), pour des entiers premiers $q_{1}, \ldots, q_{u}$ et $q_{1}^{\prime}, \ldots, q_{v}^{\prime}$ et un entier $t$, fixés, il existe une infinité d'extensions quadratiques $k / \mathbf{Q}$ dans lesquelles les $q_{i}$ sont ramifiés, les $q_{i}^{\prime}$ inertes et telles que $t|| C l_{k} \mid$. Ceci s'applique de plusieurs façons ici : il suffit de décomposer $\left\{p_{1}, \ldots, p_{r}\right\}=S_{1} \cup S_{2}$ en deux sousensembles disjoints (l'un d'entre eux pouvant être vide), et de choisir $t$ impair $>\sup \left(2 s_{1}-1, s_{2}^{2}-s_{2}+1\right)$ où $s_{1}=\sup S_{1}$ et $s_{2}=\sup S_{2}$. Il y a alors une infinité de $k / \mathrm{Q}$ quadratiques dans lesquelles les $p$ de $S_{1}$ se ramifient et ceux de $S_{2}$ restent inertes et telles que $t|m=| C l_{k}^{\prime} \mid$. D'où par la proposition, une infinité de $K$ avec $p_{1}, \ldots, p_{r}$ divisant $i(K)$, c'est à dire $n \mid i(K)$.

\section{Appendice :}

La méthode de M. Bauer par le polygone de Newton.

On se donne un entier premier $p$ et un polynôme unitaire

$$
P=X^{n}+c_{1} X^{n-1}+\cdots+c_{n-1} X+c_{n} \in \mathbf{Z}[X]
$$

de racines $\omega=\omega_{1}, \omega_{2}, \ldots, \omega_{n}$ (non nécessairement distinctes) dans une clôture algébrique de $\mathbf{Q}$. D'où l'extension de corps

$$
K=\mathbf{Q}\left(\omega_{1}, \ldots, \omega_{n}\right) \supset k=\mathbf{Q}(\omega) \supset \mathbf{Q} .
$$


On choisit $\mathcal{B} \mid p$ dans $\mathcal{O}_{K}$ et l'on pose $p=\mathcal{B} \cap \mathcal{O}_{k} ;$ on a donc des décompositions de la forme

$$
p \mathcal{O}_{k}=p^{e p} \ldots \quad \text { et } \quad p \mathcal{O}_{K}=\mathcal{B}^{e_{\mathcal{B}}} \ldots
$$

Comme $K / \mathbf{Q}$ est galoisienne, $e=e_{\mathcal{B}}$ ne dépend pas de $\mathcal{B}$ mais seulement de $p$. Soit $w=w_{\mathcal{B}}$ la valuation associée à $\mathcal{B}$ et $v=v_{p}$ la valuation $p$-adique de $\mathbf{Z}$; on suppose les racines ordonnées par ordre croissant de valuation dans $\mathcal{O}_{K}$ :

$$
w(\cdot): \underbrace{\omega_{1}, \ldots, \omega_{k_{1}}}_{a_{1}}, \underbrace{\omega_{k+1}, \ldots, \omega_{k_{1}+k_{2}}}_{a_{2}}, \cdots
$$

Comme les coefficients $c_{j}$ vérifient

$$
c_{j}= \pm \sum_{i \leq i_{1}<\cdots<i_{j} \leq n} w_{i_{1}} \ldots w_{i_{j}}
$$

on en déduit

$$
\begin{aligned}
1 \leq j & \leq k_{1} \Longrightarrow w\left(c_{j}\right) \geq j a_{1} \Longrightarrow v\left(c_{j}\right) \geq \frac{j a_{1}}{e} \\
w\left(c k_{1}\right) & =k_{1} a_{1} \Longrightarrow v\left(c_{k_{1}}\right)=\frac{k_{1} a_{1}}{e}\left(\Longrightarrow e \mid k_{1} a_{1}\right)
\end{aligned}
$$

de mème

$$
\begin{aligned}
k_{1}+1 \leq j \leq k_{1}+k_{2} \Longrightarrow v\left(c_{j}\right) \geq & \left(k_{1} a_{1}+\left(j-k_{1}\right) a_{2}\right) / e \\
v\left(c_{k_{1}+k_{2}}\right)=\frac{k_{1} a_{1}}{e}+\frac{k_{2} a_{2}}{e} & \left(\Longrightarrow e \mid k_{2} a_{2}\right)
\end{aligned}
$$

etc...

D'où le polygone de Newton pour $P$ et $p(=$ bord inférieur de l'enveloppe convexe des points $\left(i, v\left(c_{i}\right)\right)$, avec $\left.c_{0}=1\right)$ : 


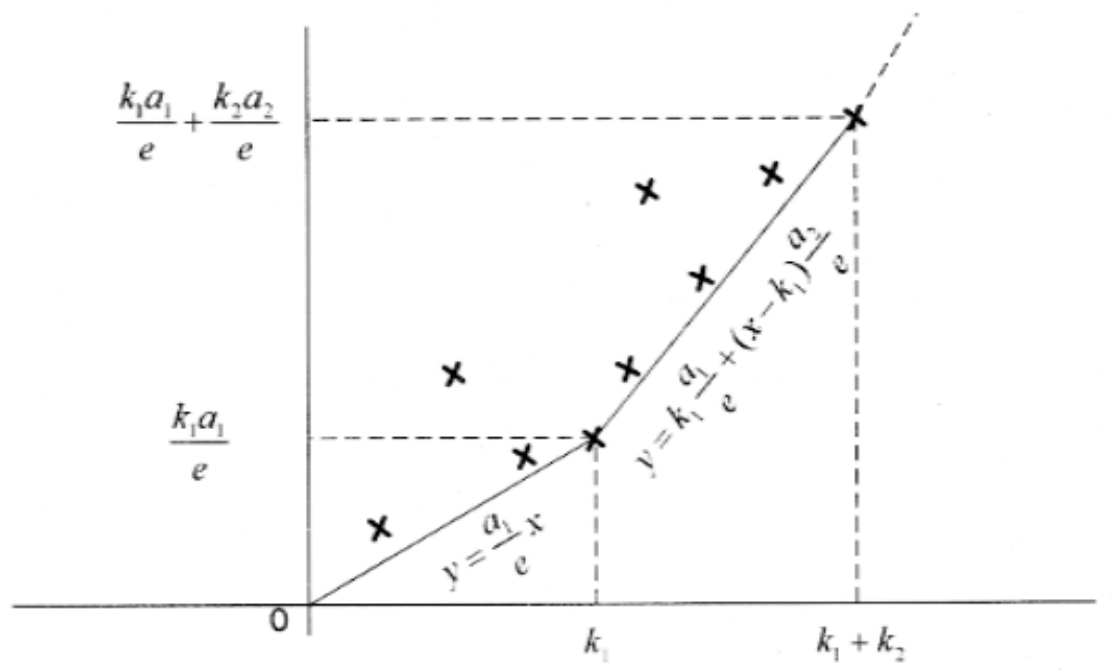

On pose $\alpha_{i}=\frac{k_{i} a_{i}}{e}(\in \mathbf{N})$.

Les pentes du polygone de Newton, $\frac{\alpha_{i}}{k_{i}}=\frac{a_{i}}{e}$ s'appellent les nombres de Puiseux (de $P$ et $p$ ). Notons que $\omega=\omega_{1}$ se décompose sous la forme $\omega \mathcal{O}_{K}=\mathcal{B}^{a_{\mathcal{B}}} \ldots$ (où $a_{\mathcal{B}}=a_{1}$ ) et $\omega \mathcal{O}_{k}=p^{a} p \ldots$ et alors

$$
\frac{a_{p}}{e_{p}}=\frac{a_{\mathcal{B}}}{e} \quad \forall \mathcal{B}|p| p
$$

\section{Applications}

* Si les nombres de Puiseux sont $\frac{\alpha_{1}}{1}, \ldots, \frac{\alpha_{n}}{1}$, on en déduit que toutes les racines sont de valuation $w$ distincte, donc que le groupe de décomposition $D_{\mathcal{B}}$ laisse chaque $\omega_{i}$ fixe et est donc trivial ; par conséquent $p$ se décompose totalement dans $\mathcal{O}_{k}$ et $\mathcal{O}_{K}$.

En particulier, si $p<n$, alors $p \mid i(k)$ (par le corollaire 2).

** S'il n'y a qu'un nombre de Puiseux $\frac{\alpha}{n}$ avec $(\alpha, n)=1$, alors toutes les racines $\omega_{i}$ ont même valuation $w(\omega)=a$ et

$$
\frac{a_{p}}{e_{p}}=\frac{\alpha}{n} \Rightarrow n\left|e_{p} \alpha \Rightarrow n\right| e_{p} \Rightarrow n=e_{p} \quad\left(\operatorname{car} e_{p} \leq[k: \mathbf{Q}] \leq n\right)
$$


$\Rightarrow P$ irréductible (c'est le polynôme minimal de $\omega$ sur $\mathbf{Q}$ ) et $p \mathcal{O}_{k}=p^{n}$ est totalement ramifié dans $\mathcal{O}_{k}$; donc $p \nmid i(K)$.

*** On suppose $P$ irréductible (en lui imposant par exemple la condition ** avec $q$ à la place de $p$, pour un $q$ premier $\neq p$ ).

Si les nombres de Puiseux pour $p$ (et $P$ ) sont $\frac{\alpha_{1}}{2}, \frac{\alpha_{2}}{1}, \ldots, \frac{\alpha_{n-1}}{1}$ avec $\alpha_{1}$ impair, alors $\frac{\alpha_{1}}{2}=\frac{a_{1}}{e} \Rightarrow 2 \mid e$

et $p \mathcal{O}_{k}=p_{1}^{2} p_{2} \ldots p_{n-1}$ est la forme de la décomposition de $p$ dans $\mathcal{O}_{k}$.

(Car seules $\omega_{1}$ et $\omega_{2}$ peuvent être racines dans $K_{\mathcal{B}}$ d'un même polynôme irréductible $\in \mathbf{Q}_{p}[X]$ et elles le sont effectivement car sinon $p$ serait non ramifié dans $K$ et ceci contredirait $2 \mid e)$.

En particulier, si $p<n-1$, alors $p \mid i(k)$ (et aussi $\operatorname{disc}(k / \mathbf{Q})$ ).

Pour d'autres exemples, on pourra consulter [B].

\section{References}

[B] M. BAUER Über die außerwesentlichen Diskriminantenteiler einer Gattung Math. Ann. 64, pp. 572 - 576, (1907).

[H] H. HASSE Number theory, Grundlehren der math. wiss., № 29, Springer-Verlag (1980).

[N] W. NARKIEWICZ "Elementary and analytic theory of algebraic numbers" 2nd edition, Springer Verlag (1990).

[Y] Y. YAMAMOTO On unramified Galois extensions of quadratic number fields, Osaka J. Math. 7, pp. 57 - 76, (1970).

Received by : December 20, 1999

\section{Denis Hémard}

Université de Valenciennes et du Hainaut Cambrésis

Laboratoire LAMATH B.P. 311

59304 Valenciennes Cedex

France 